\title{
Vector Flow Imaging Compared with Conventional Doppler Ultrasound and Thermodilution for Estimation of Blood Flow in the Ascending Aorta
}

\begin{abstract}
Hansen, Kristoffer Lindskov; Møller-Sørensen, Hasse; Kjaergaard, Jesper; Jensen, Maiken Brit; Lund, Jens Teglgaard; Pedersen, Mads Møller; Olesen, Jacob Bjerring; Jensen, Jørgen Arendt; Bachmann Nielsen, Michael
\end{abstract}

Published in:

Ultrasonic Imaging

Link to article, DOI:

$10.1177 / 0161734615620137$

Publication date:

2017

Document Version

Publisher's PDF, also known as Version of record

Link back to DTU Orbit

Citation (APA):

Hansen, K. L., Møller-Sørensen, H., Kjaergaard, J., Jensen, M. B., Lund, J. T., Pedersen, M. M., Olesen, J. B., Jensen, J. A., \& Bachmann Nielsen, M. (2017). Vector Flow Imaging Compared with Conventional Doppler Ultrasound and Thermodilution for Estimation of Blood Flow in the Ascending Aorta. Ultrasonic Imaging, 39(1), 3-18. https://doi.org/10.1177/0161734615620137

\section{General rights}

Copyright and moral rights for the publications made accessible in the public portal are retained by the authors and/or other copyright owners and it is a condition of accessing publications that users recognise and abide by the legal requirements associated with these rights.

- Users may download and print one copy of any publication from the public portal for the purpose of private study or research.

- You may not further distribute the material or use it for any profit-making activity or commercial gain

- You may freely distribute the URL identifying the publication in the public portal 


\section{Vector Flow Imaging Compared with Conventional Doppler Ultrasound and Thermodilution for Estimation of Blood Flow in the Ascending Aorta}

Ultrasonic Imaging

$1-16$

(C) The Author(s) 2015

Reprints and permissions: sagepub.com/journalsPermissions.nav DOI: $10.1177 / 0161734615620137$ ultrasonicimaging.sagepub.com

@SAGE

\author{
Kristoffer Lindskov Hansen', Hasse Møller-Sørensen², \\ Jesper Kjaergaard ${ }^{3}$, Maiken Brit Jensen², Jens Teglgaard Lund ${ }^{4}$, \\ Mads Møller Pedersen', Jacob Bjerring Olesen ${ }^{5}$, \\ Jørgen Arendt Jensen ${ }^{\text {, }}$ and Michael Bachmann Nielsen'
}

\begin{abstract}
Transverse oscillation (TO) is a real-time ultrasound vector flow method implemented on a commercial scanner. The TO setup was examined on a flowrig with constant and pulsatile flow. Subsequently, 25 patients undergoing cardiac bypass surgery were scanned intraoperatively with TO on the ascending aorta and compared to transesophageal echocardiography (TEE) and pulmonary artery catheter thermodilution (PACTD). On the flowrig, TO had a precision of $5.5 \%, 9.4 \%$ and $14.7 \%$, a percentage error of $18.2 \%, 14.6 \%$ and $40.7 \%$, and a mean bias of 0.4 $\mathrm{cm} / \mathrm{s}, 36.8 \mathrm{ml} / \mathrm{min}$ and $32.4 \mathrm{ml} / \mathrm{min}$ for velocity and flow rate (constant and pulsatile) estimation. The correlation coefficients for all flowrig evaluations were 0.99 indicating systematic bias. After bias correction, the percentage error was reduced to $11.5 \%, 12.6 \%$ and $15.9 \%$ for velocity and flow rate (constant and pulsatile) estimation. In the in vivo setup, TO, TEE, and PACTD had a precision of $21.9 \%, 13.7 \%$, and $12.0 \%$. TO compared with TEE and PACTD had a mean bias of $12.6 \mathrm{~cm} / \mathrm{s}$ and $-0.08 \mathrm{l} / \mathrm{min}$, and a percentage error of $23.4 \%$, and $36.7 \%$, respectively. The percentage error was reduced to $22.9 \%$ for the TEE comparison, but increased to $43.8 \%$ for the PACTD comparison, after correction for the systematic bias found in the flowrig. TO is a reliable and precise method for velocity and flow rate estimation on a flowrig. However, TO with the present setup, is not interchangeable with PACTD for cardiac volume flow estimation, but is a reliable and precise angle-independent ultrasound alternative for velocity estimation of cardiac flow.
\end{abstract}

\footnotetext{
'Department of Radiology, Rigshospitalet, Copenhagen University Hospital, Copenhagen, Denmark 2Department of Cardiothoracic Anesthesiology, Rigshospitalet, Copenhagen University Hospital, Copenhagen, Denmark

${ }^{3}$ Department of Cardiology, Rigshospitalet, Copenhagen University Hospital, Copenhagen, Denmark ${ }^{4}$ Department of Cardiothoracic Surgery, Rigshospitalet, Copenhagen University Hospital, Copenhagen, Denmark ${ }^{5}$ Center for Fast Ultrasound Imaging, DTU Elektro, Technical University of Denmark, Kongens Lyngby, Denmark

Corresponding Author:

Kristoffer Lindskov Hansen, Department of Radiology, Rigshospitalet, Copenhagen University Hospital, Blegdamsvej 9, 2100 Copenhagen, Denmark.

Email: lindskov@gmail.com
} 


\section{Keywords}

vector flow imaging, transverse oscillation, flowrig, intraoperative cardiac ultrasound, ascending aorta

\section{Introduction}

Cardiac flow is often monitored during major surgery. Two of the most commonly used methods are transesophageal echocardiography (TEE) and pulmonary artery catheter thermodilution (PACTD).

TEE has achieved a major role in perioperative echocardiographic assessment of cardiac function and is widely used as a perioperative monitoring tool during cardiac surgery. ${ }^{1}$ TEE is used to assess the cardiac structural and functional anatomy, and by applying spectral Doppler ultrasound (US), estimation of blood flow velocities, velocity ratios, and ejection phase indices can be obtained. ${ }^{2}$ However, the angle dependency in conventional spectral Doppler US reduces the reliability and the precision of the measurements. ${ }^{3}$

PACTD is recommended for monitoring of cardiac function during surgical procedures where hemodynamic changes are foreseen, and the method provides measures of cardiac output. ${ }^{4}$ PACTD is an invasive method with a small risk of complications such as pulmonary embolism and hemorrhage. ${ }^{5}$

Different methods have been proposed in the last decades to circumvent the limitation of angle dependency in conventional ultrasound Doppler systems to achieve vector velocity estimation. ${ }^{6-9}$ The vector velocity methods can be divided into two groups of approaches. One solution is to measure the blood motion from two different angles in either transmit or receive. ${ }^{7}$ Tortoli et al. have recently published a paper where two different approaches using dual focused beams and dual plane waves were compared with conventional spectral Doppler indicating that vector flow is superior to the reference method. ${ }^{10}$ The other solution for vector velocity estimation is to track speckle motion as proposed by Trahey et al. ${ }^{9}$ Recent studies using speckle tracking include Nyrnes et al., visualizing in arterial septal defects, and Lovstakken et al., reporting on intraoperative assessment on blood flow patterns in coronary anastomoses and in congenital heart disease. ${ }^{11-13}$ One of the main advantages of the speckle tracking approaches is a potential better performance in depth compared with the dual beam approaches, as no beam crossing is necessary. However, the computational demands are often higher than the dual beam approaches, thus, implementation of speckle tracking in real time is cumbersome.

A novel US method for blood flow measurement using a dual receive scheme is transverse oscillation (TO). The TO method, introduced by Jensen and Munk, estimates the in-plane blood flow, angle-independently and in real time. ${ }^{14,15}$ TO has been tested in computer simulations and with flow phantoms, ${ }^{16}$ validated in vivo against magnetic resonance imaging (MRI) angiography ${ }^{17,18}$ and against conventional spectral Doppler US. ${ }^{19}$ Also, TO has been used in estimation of tissue motion as reported by Liebgott et al. and Varray et al. ${ }^{20,21}$ Preliminary results on epicardiac intraoperative US scans with TO have been published, however, in these studies, only a few patients were examined. ${ }^{22,23}$ The previous studies of the aortic flow indicated that vector velocities obtained with TO are biased when compared with estimates of TEE and PACTD. To confirm these results and to investigate the different contributing factors to the bias, a larger study including both flowrig measurements and in vivo examination of 25 patients was performed.

TO implemented on a commercial scanner (ProFocus 2202 UltraView, BK Medical, Herlev, Denmark) with a linear transducer (8670, BK Medical, Herlev, Denmark) was examined on a flowrig with constant and pulsatile flow for flow velocity and flow rate estimation. Subsequently, 25 patients were scanned intraoperatively with TO to estimate the blood flow of the ascending aorta. The systolic velocities and the cardiac output derived from vector velocities were compared with estimates obtained with TEE and PACTD. 


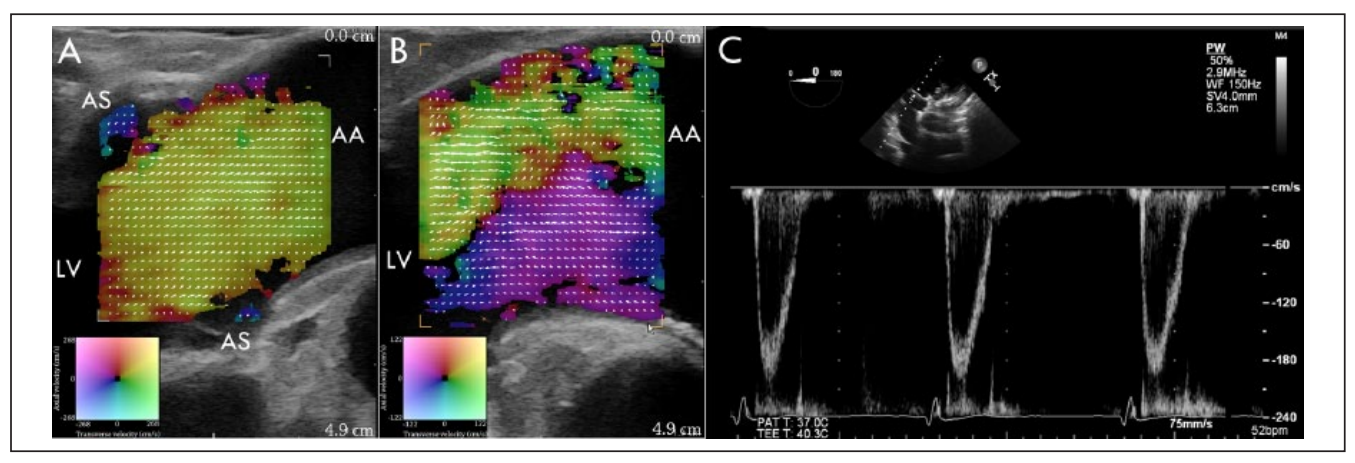

Figure I. Systolic vector velocities in the ascending aorta of two patients. In A, antegrade flow is filling the aorta along with vortices in the aortic sinuses. In $\mathrm{B}$, a substantial systolic backflow is present along the convex vessel wall. In $C$, the corresponding TEE measurement of the patient in $A$ is shown. TEE = transesophageal echocardiography; $L V=$ left ventricle; $A A=$ ascending aorta; $A S=$ aortic sinus.

The aims of the study were to find the precision, the percentage error, and the systematic bias of TO on a flowrig, and with the in vivo data to compare the precision between TO, TEE, and PACTD, and to assess the agreement of TO corrected for the systematic bias with TEE and PACTD.

\section{Method}

The study was approved by the local ethics committee (no. H-2-2012-039). In total, 25 patients (20 males, 5 females, mean age: 65.6 years, range $41-80$ years) without any history of valvular disease and undergoing coronary bypass surgery entered the study after written informed consent.

\section{Transverse Oscillation}

TO estimates the vector velocity by emitting a conventional pulse for Doppler US. Where conventional Doppler US only estimates the axial velocity, the TO approach estimates both the axial and the transverse velocity to calculate the vector velocity. The motion in the axial direction is found as in conventional Doppler US, while the motion in the transverse direction is found by changing the sensitivity of the receiving elements and by using a special estimator. More thorough descriptions of the TO estimation scheme of vector velocities are provided by Jensen, Munk, and Udesen. ${ }^{14,16}$

A conventional US scanner (ProFocus 2202 UltraView, BK Medical, Herlev, Denmark) and a $5 \mathrm{MHz}$ linear transducer (8670, BK Medical, Herlev, Denmark) were used to obtain the vector velocity data. The vector velocities are displayed in real time on the B-mode image as colored pixels given by a two-dimensional (2D) color wheel, and furthermore, shown as small arrows superimposed on the color map (Figure 1). The colors define both flow direction and velocity magnitude, while the arrows are provided to facilitate the overall flow interpretation. The temporal resolution of the TO estimation is 16 frames/s, and the maximum scan depth is approximately $5 \mathrm{~cm}$ due to the transducer setup available. The color box was adjusted to cover the entire vessel in the axial direction and to be as wide as possible in the transverse direction, although only the central vector velocity lines, corresponding to $4 \mathrm{~mm}$ in the transverse direction, were used for the estimation of the vector velocities for the comparisons with TEE and PACTD. This is identical to a setup previously used by Hansen et al. ${ }^{24}$ More flow information is available in the vector flow 
sequences as seen in Figure 1. However, to avoid a bias in the flow estimation of a bending vessel with a centerline out-of-plane, only the central part of the color box was used for the measurements.

The vector flow estimation is displayed in real time, but no quantification of velocities is available on the scanner. Hence, the scan sequences were analyzed off-line using Matlab (Mathworks, Natick, Massachusetts) as described by Pedersen et al. ${ }^{19}$ Volume flow $\mathrm{TO}_{\mathrm{Q}}$ from vector velocities is found using two different approaches:

$$
\begin{aligned}
& \mathrm{TO}_{\mathrm{Q}_{\text {_Vmax }}}=0.5 \times \mathrm{Vmax} \times \mathrm{A}, \\
& \mathrm{TO}_{\mathrm{Q}_{\text {_Vmean }}}=\mathrm{Vmean} \times \mathrm{A},
\end{aligned}
$$

where A is the cross-sectional area of the vessel and calculated from perpendicular diameters of the vessel measured in short axis view. Vmax is the maximum velocity and Vmean is the mean velocity averaged over the selected central lines of the color box to each frame. The volume flow is calculated for every frame, and $\mathrm{TO}_{\mathrm{Q}}$ is given as a mean over each sequence. The flow is assumed parabolic and axisymmetrical for the calculation of $\mathrm{TO}_{\mathrm{Q}_{\mathrm{V}} \max }$, while only the assumption of axisymmetrical flow is made for the calculation of $\mathrm{TO}_{\mathrm{Q}_{-} \text {Vmean }}$. Further details on the volume flow calculation from vector estimates are given by Hansen et al. ${ }^{24}$

\section{Flow Phantom Setup}

A flow system (CompuFlow 1000, Shelley Medical Imaging Technologies, Toronto, Ontario, Canada) circulates a blood-mimicking fluid (BMF-US, Shelley Medical Imaging Technologies, Toronto, Ontario, Canada) in the closed-loop circuit. The linear transducer was fixated with a distance of $2 \mathrm{~cm}$ from a vessel of $0.8 \mathrm{~cm}$ in diameter with an insonation angle of $90^{\circ}$. According to previous flowrig TO experiments, the beam-to-flow angle is of less importance when using 5 $\mathrm{MHz}$ center frequency TO emission, thus, only $90^{\circ}$ insonation angle was investigated. ${ }^{25}$

TO was evaluated for increasing flow rates with both constant and pulsatile flow. Vector velocities at each flow rate setting were recorded twice to assess the precision of TO. At one flow setting for both constant $(18 \mathrm{ml} / \mathrm{s})$ and pulsatile flow $(16 \mathrm{ml} / \mathrm{s})$, the standard deviation $(S D)$ of TO was estimated with 10 repeated measurements. The flow rates for $S D$ measurements were selected as approximately the half of the maximum flow rates used for constant $(26 \mathrm{ml} / \mathrm{s})$ and pulsatile (34 $\mathrm{ml} / \mathrm{s}$ ) flow in the flowrig. The size of the color box, Doppler gain, and pulse repetition frequency (PRF) were adjusted before each scan sequence of $15 \mathrm{~s}$ of recording. The PRF was increased from $0.4 \mathrm{kHz}$ to $5.1 \mathrm{kHz}$ and $0.2 \mathrm{kHz}$ to $4.1 \mathrm{kHz}$ in the experiments with constant and pulsatile flow, respectively, while the average gain was $58.6 \%(S D=2.8 \%)$ for constant and $56.6 \%(S D=$ $2.9 \%$ ) for pulsatile flow.

\section{In Vivo Setup}

TO was used intraoperatively under sterile settings to record epiaortic scan sequences after standard sternotomy, as described previously. ${ }^{23}$ The ascending aorta was scanned along the long axis (Figure 1), and to enhance the acoustic transmission from the probe to the aortic surface, warm sterile saline was poured into the mediastinal cavity as recommended by the American Society of Echocardiography and the Society of Cardiovascular Anesthesiologists. ${ }^{26}$ The blood flow was estimated twice for each patient, and for each scan sequence, the size of the color box, Doppler gain, and PRF were adjusted. The PRF was on average $10.04 \mathrm{kHz}(S D=1.1 \mathrm{kHz})$ and the 
Doppler gain on average $51.8 \%(S D=4.7 \%)$. The highest velocities, measurable with TO implemented on the commercial scanner from BK Medical, are around $200 \mathrm{~cm} / \mathrm{s}^{23}$ These high velocities were not reached in this study, thus, aliasing did not occur. The segment of the ascending aorta investigated with TO was governed by the accessibility to the aorta mainly depending on the dimensions of the sternotomy. The transducer was placed as proximal on the ascending aorta as possible without including the aortic valves. The average beam-to-flow angle was $73.6^{\circ}(S D$ $=8.6^{\circ}$ ). Each scan sequence of $15 \mathrm{~s}$ of recording corresponded on average to 17.5 (range: 14-23) heart cycles.

\section{Transesophageal Echocardiography and Pulmonary Artery Catheter Thermodilution}

TEE scans were performed with a Philips X7-2t transducer on a Philips iE33 scanner (Philips Healthcare, Eindhoven, the Netherlands) using pulsed wave spectral Doppler US. The scan parameters were adjusted for flow imaging, and the scan sequences were obtained through the aortic valve aperture in the deep transgastric long axis view with the range gate placed in the ascending aorta corresponding to the location of the compared TO estimation.

PACTD was performed with a 7.5F pulmonary artery catheter (Swan-Ganz Oximetry TD catheter, Baxter Edwards Critical Care, Irvine, California) and the cardiac output was determined by the HP-Philips M1012A Cardiac Output Module (Hewlett Packard, Boeblingen, Germany) as a mean of four bolus injections of $10 \mathrm{ml}$ saline. For both TEE and PACTD estimations, each patient was subjected to two measurements to assess precision of the methods.

Epicardiac scan sequences of the blood flow in the ascending aorta were recorded with TO after standard sternotomy and before cannulation for extracorporeal circulation. Standard spectral Doppler measurements of blood flow in the ascending aorta were obtained with TEE, and cardiac output measurements were obtained with PACTD. For each patient, TO and TEE measurements were acquired within $5 \mathrm{~min}$, while PACTD measurements were obtained simultaneously with the TO measurements.

\section{Statistical Analyses}

The peak velocity and the flow rate were found with TO on a flowrig with constant and pulsatile flow. In the in vivo setup, peak systolic velocities were obtained with TO and TEE, and cardiac output was determined from vector velocities of TO as well as measured by PACTD. For both constant and pulsatile flow on the flowrig, the $S D$ was found with 10 repeated measurements. The precision of the flowrig measurements for TO and of the in vivo measurements for TO, TEE, and PACTD were determined by replicate measurements. The precision $P$ corresponded to two $S D$ of the difference between replicate measurements $a$ and $b$ of a method $x$, divided by the mean, and given in percentage:

$$
P=\frac{2 \times S D\left(x_{n}^{a}-x_{n}^{b}\right)}{\bar{x}} \times 100,
$$

where $n$ is the number of replicated experiments, and $\bar{x}$ is the average value obtained. Percentage error $(P E)$ for each comparison of two methods $x$ and $y$ was calculated just as the precision for replicate measurements, that is, two $S D$ of the mean difference divided by the mean of the two methods and given in percentage: 
Table I. Precision Estimates of TO in Flowrig and of TO, TEE, and PACTD In Vivo along with SD of TO in Flowrig.

\begin{tabular}{|c|c|c|}
\hline & $\begin{array}{l}\text { Precision } P \text { of Replicate } \\
\text { Measurements (\%) }\end{array}$ & $\begin{array}{l}\text { Mean/SD over } 10 \\
\text { Repeated Estimations (SD } \\
\text { Given in \% of Mean) }\end{array}$ \\
\hline \multicolumn{3}{|l|}{ Constant flow in flowrig } \\
\hline $\mathrm{TO}_{\mathrm{Vel}}(\mathrm{cm} / \mathrm{s})$ & 5.5 & $65.7 / 1.0(1.4 \%)$ \\
\hline $\mathrm{TO}_{\mathrm{Q}_{\mathbf{V} \max }}(\mathrm{ml} / \mathrm{min})$ & 13.0 & $738.2 / 9.9(1.3 \%)$ \\
\hline $\mathrm{TO}_{\mathrm{Q} \_ \text {Vmean }}(\mathrm{ml} / \mathrm{min})$ & 9.4 & $889.6 / 11.0(1.2 \%)$ \\
\hline \multicolumn{3}{|l|}{ Pulsatile flow in flowrig } \\
\hline 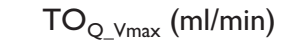 & 16.2 & $86.9 / 2.4(2.8 \%)$ \\
\hline $\mathrm{TO}_{\mathrm{V}_{\text {mean }}}(\mathrm{ml} / \mathrm{min})$ & 14.7 & $101.0 / 4.2(4.2 \%)$ \\
\hline \multicolumn{3}{|l|}{ Ascending aorta in vivo } \\
\hline $\mathrm{TO}(\mathrm{cm} / \mathrm{s})$ & 21.9 & $N / A$ \\
\hline $\mathrm{TO}_{\mathrm{Q} \__{\max }}(1 / \mathrm{min})$ & 24.8 & N/A \\
\hline $\mathrm{TO}_{\mathrm{Q} \_ \text {Vmean }}(1 / \mathrm{min})$ & 88.1 & $\mathrm{~N} / \mathrm{A}$ \\
\hline TEE $(\mathrm{cm} / \mathrm{s})$ & 13.7 & $\mathrm{~N} / \mathrm{A}$ \\
\hline PACTD (I/min) & 12.0 & N/A \\
\hline
\end{tabular}

$\mathrm{TO}=$ transverse oscillation; TEE = transesophageal echocardiography; PACTD = pulmonary artery catheter thermodilution; $S D=$ standard deviation.

$$
P E=\frac{2 \times S D\left(x_{n}-y_{n}\right)}{(\bar{x}+\bar{y}) / 2} \times 100,
$$

where $n$ is the number of replicated experiments, and $\bar{x}$ and $\bar{y}$ are the average values obtained for method $x$ and $y$. Thus, the precision $P$ and percentage error $P E$ are going toward 0 for perfectly replicated measurements and perfect comparisons, respectively. Comparisons were done with Bland-Altman and linear regression analyses. From the linear regression analyses of the flowrig data, the systematic bias for both velocity and flow rate estimation was calculated and applied to data to calculate a corrected percentage error. Student's $t$ test was applied to data and $p<0.05$ considered significant. Statistical analyses were performed with MATLAB and SPSS (SPSS Inc., Chicago, Illinois).

\section{Results}

In Table 1 are given the precision of TO in flowrig, and of TO, TEE, and PACTD in vivo. For flowrig measurements, the $S D$ for 10 repeated measurements is given in percentage.

The precision of TO in flowrig was not different for flow rate estimation using Vmax and Vmean for constant $(p<0.18)$ and pulsatile flow $(p<0.97)$. Furthermore, when changing from constant to pulsatile flow, no difference was found in precision for TO for flow rate estimation using Vmax $(p<0.32)$ and Vmean $(p<0.35)$. Comparing the precision of TO in flowrig with the precision of TO in vivo showed a difference in velocity $(p<0.005)$ and in flow rate estimation (Vmax: $p<0.001$, Vmean: $p<0.001$ ).

For the in vivo setup, the precision was not different for TO compared with TEE $(p<0.25)$ but different for TO compared with PACTD using Vmax $(p<0.001)$ and Vmean $(p<0.001)$.

The mean differences with limits of agreement (LOA) and confidence intervals (CI) for TO compared with flowrig, TEE, and PACTD as well as the correlation coefficients are listed in Table 2. 


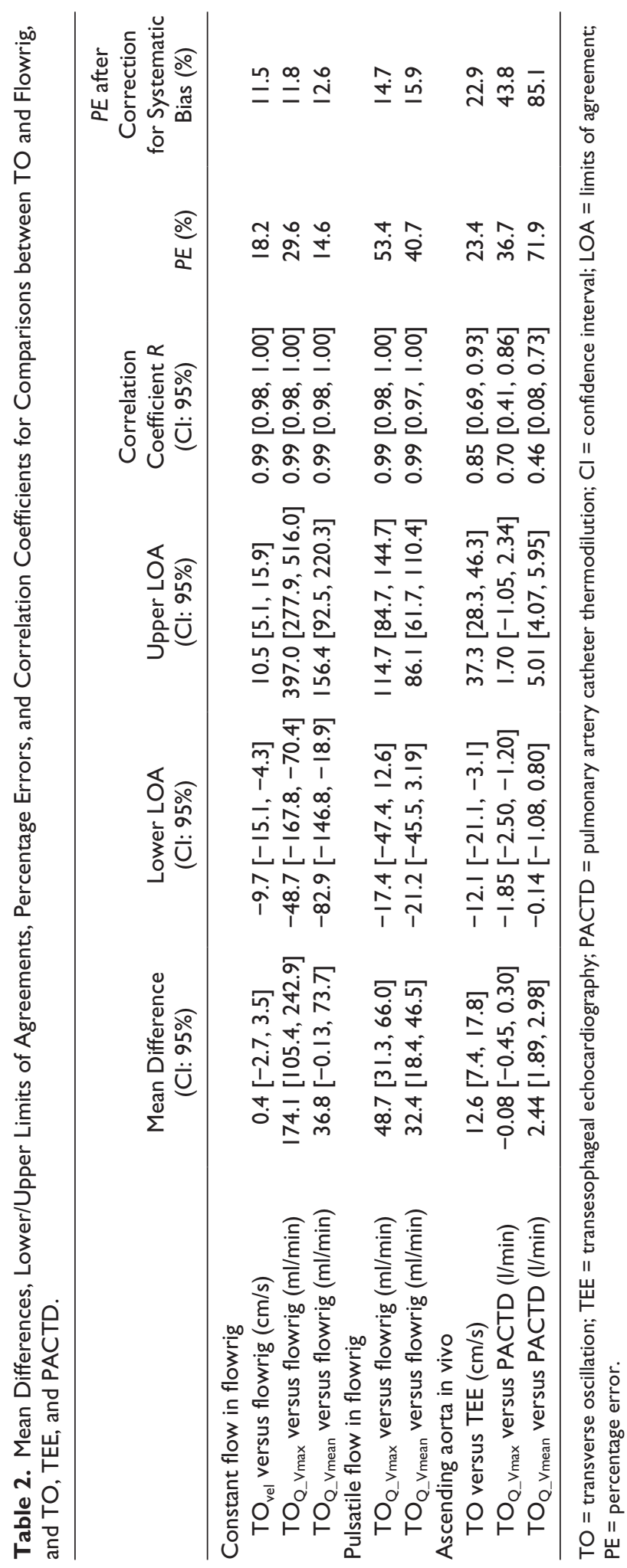


A systematic bias was present for TO measurements in the flowrig for both constant and pulsatile flow as seen in Figures 2 to 4 and Table 2. Thus, for each comparison listed in Table 2, the percentage error is given before and after correction of the TO measurements with the systematic bias found in the flowrig experiments. In vivo TO data are corrected with the systematic bias found from the TO measurements obtained in the flowrig. For the comparison with PACTD, the systematic bias found in the flowrig with pulsatile flow is used for the correction.

The average percentage error is improved when applying the correction for the flowrig experiments $(p<0.05)$, but not for the in vivo experiments $(p<0.24)$. The percentage error for flow rate estimation without correction is increased on the flowrig from constant to pulsatile flow (Vmax: $p<0.001$; Vmean: $p<0.006$ ), but with the correction applied, the difference is not significant (Vmax: $p<0.34$; Vmean: $p<0.33$ ). Comparing flowrig and in vivo measurements for flow rate estimation showed increased percentage errors whether correction for systematic bias was applied or not (Vmax: $p<0.001$; Vmean: $p<0.001$ ). Comparing flowrig and in vivo measurements for flow velocity showed equally good performance without correction $(p<0.98)$, but increased percentage error from flowrig to in vivo, when correction was performed $(p<0.03)$. Bland-Altman and regression plots for all comparisons are shown in Figures 2 to 6.

\section{Discussion}

TO has previously been validated with MRI, ultrasound dilution technique (UDT), and spectral Doppler. ${ }^{17-19,24}$ The TO method implemented on the commercial scanner has also been validated on a flowrig with constant flow, ${ }^{25}$ and studies have investigated the feasibility of TO estimation of the heart intraoperatively on a few patients. ${ }^{22,23}$ In this study, TO was evaluated in a flowrig with both constant and pulsatile flow to investigate the systematic bias of TO in a controlled setup, and to distinguish different factors contributing to the bias. Subsequently, 25 patients were scanned intraoperatively with TO on the ascending aorta and compared with estimates of TEE and PACTD to investigate how TO performed when corrected for the systematic bias found in the flowrig.

The TO method was in the flowrig shown to be both precise and robust with $S D$ of $1.2 \%$ to $1.4 \%$ and precision of $5.5 \%$ to $16.2 \%$ (Table 1). By inspecting Figures 2 to 4 and the correlation coefficients listed in Table 2, it is evident that a systematic bias is present for both flow velocity and flow rate estimation obtained with $\mathrm{TO}$ in the flowrig.

Previous studies have shown that vector velocities obtained with the TO method are underestimated by approximately $10 \%$, when compared with flowrig and conventional spectral Doppler. ${ }^{16,19}$ In this study with TO implemented on a commercial US scanner, a systematic underestimation of approximately 14\% was found for velocities above $60 \mathrm{~cm} / \mathrm{s}$ (Figure 2). This also affected the flow rate estimations on the flowrig with constant flow, where the approaches using Vmax or Vmean were underestimated with $23 \%$ and $8 \%$, respectively (Figure 3 ). The systematic underestimation with constant flow is mainly due to a bias in the estimation scheme, which can be compensated for in an optimized setup as demonstrated by Jensen. ${ }^{27}$

TO estimation of flow rate on the flowrig with pulsatile flow showed reduced precision and increased systematic bias as compared with TO estimation of flow rate on the flowrig with constant flow (Table 1 and 2). The reason is mainly the PRF setting. In velocity imaging, there is only a limited amount of measurements available per direction, which limits the lowest velocity measurable. For the vector velocity estimation in the flowrig with pulsatile flow, the PRF was adjusted to estimate the peak velocities without aliasing, and with this PRF setting, a substantial part of lower velocities were consequently missed out. The flow rate estimation schemes using Vmax and Vmean were underestimated with $40 \%$ and $31 \%$, respectively (Figure 4). However, with a correction for the systematic bias applied, no difference in percentage error for flow rate estimation using TO was found for constant and pulsatile flow on the flowrig (Table 2). 


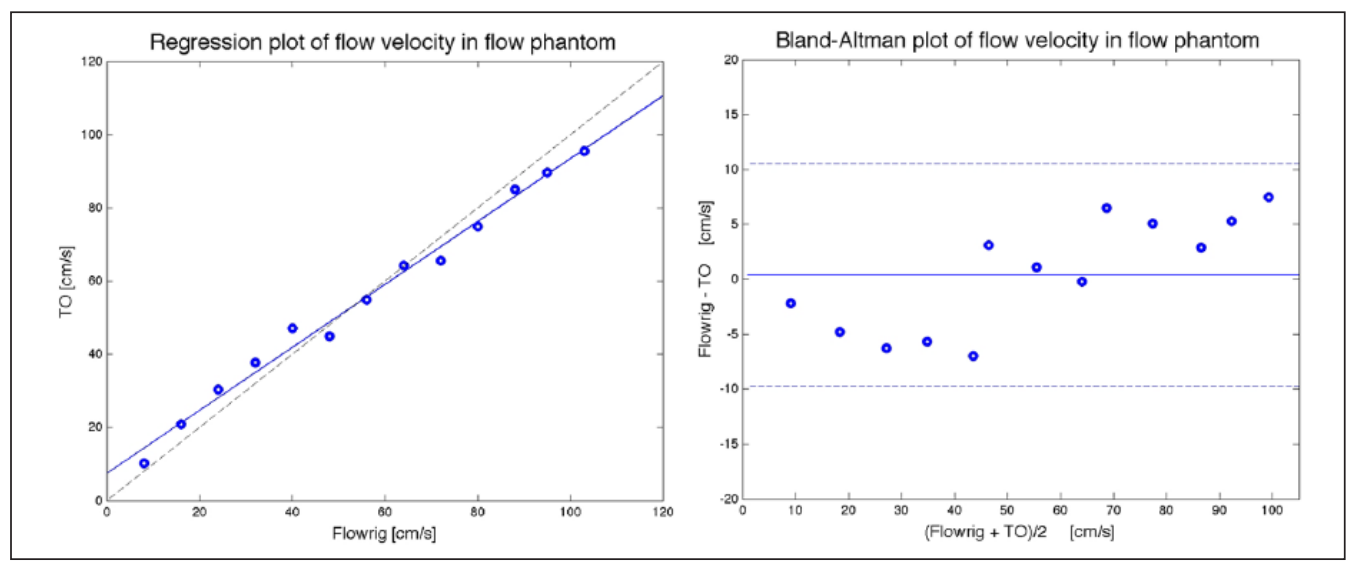

Figure 2. Flow velocity estimation with TO on the flowrig evaluated with Bland-Altman and regression plots. $\mathrm{TO}=$ transverse oscillation.

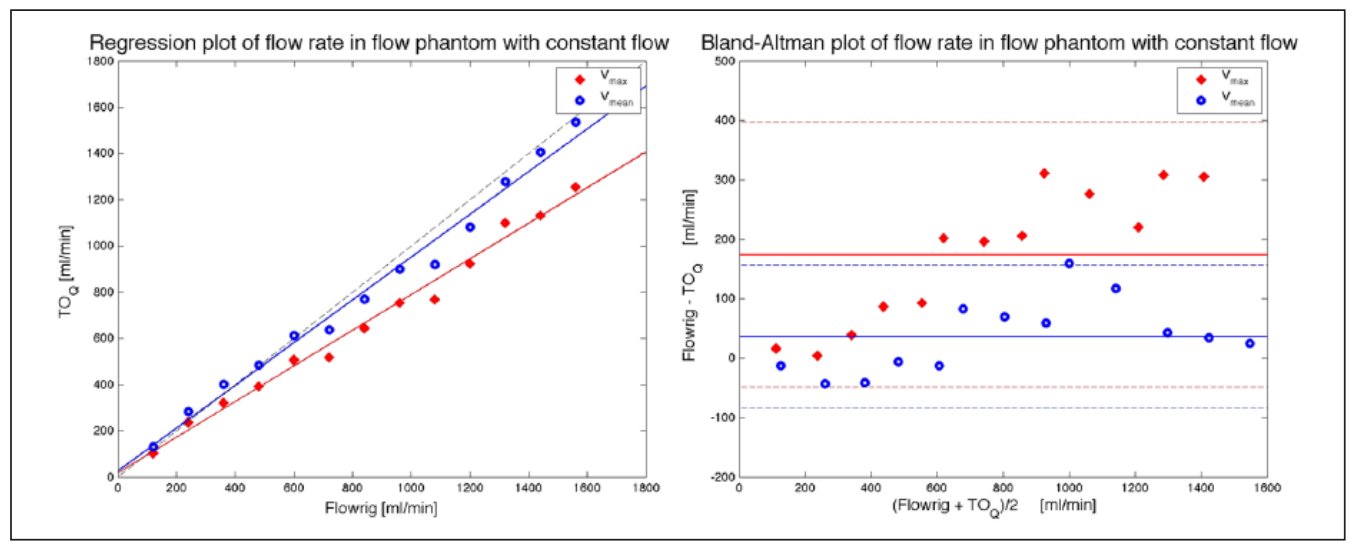

Figure 3. Flow rate estimation with TO on the flowrig with constant flow evaluated with Bland-Altman and regression plots. $\mathrm{TO}=$ transverse oscillation.

When two methods are compared, it is crucial to assess the precision of each method before conclusion is made on the interchangeability. The repeatability, that is, precision of each method, can be obtained from replicate measurements. ${ }^{28}$ In this study, TO was comparable in precision to TEE, but less precise than PACTD (Table 1). Critchley et al. have proposed that the LOA of a method comparison study should not be wider than the expected LOA calculated from the precision measures of each method. ${ }^{29}$ The expected LOA is calculated as,

$$
S D_{a+b}=\sqrt{S D_{a}^{2}+S D_{b}^{2}}
$$

where $S D$ is the standard deviation of each method $a$ and $b$. Instead of $S D$, the calculated precision is often used. ${ }^{30}$

Therefore, the expected LOA of TO compared with TEE is $25.8 \%$, while the expected LOA for $\mathrm{TO}_{\mathrm{Q}_{-} \text {max }}$ compared with PACTD is $27.6 \%$ and $\mathrm{TO}_{\mathrm{Q}_{-} \text {vmean }}$ is $88.9 \%$. According to Table 2, 


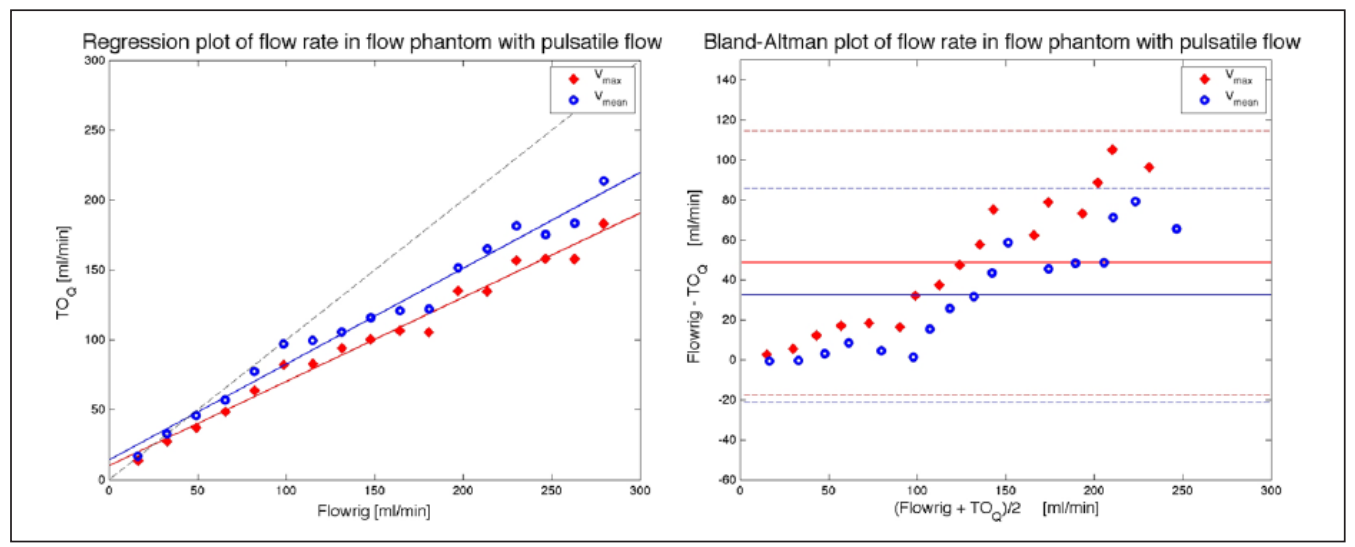

Figure 4. Flow rate estimation with TO on the flowrig with pulsatile flow evaluated with Bland-Altman and regression plots. $\mathrm{TO}=$ transverse oscillation.

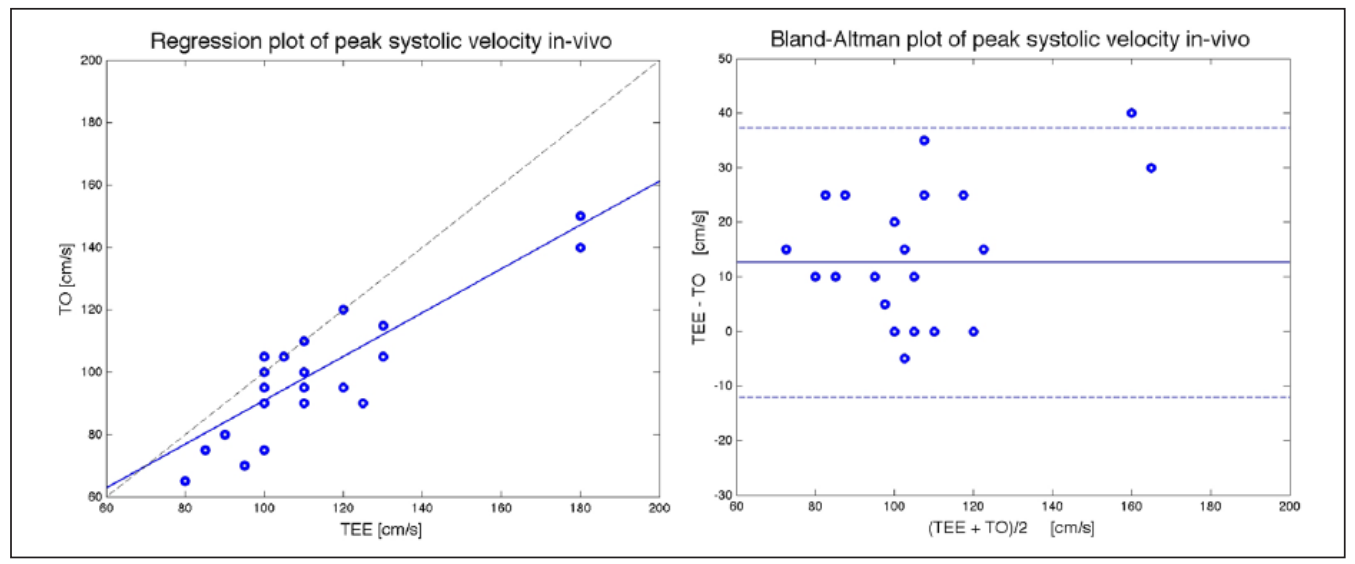

Figure 5. Peak systolic velocity estimated with TO and TEE in vivo and evaluated with Bland-Altman and regression plots. $\mathrm{TO}=$ transverse oscillation; TEE = transesophageal echocardiography.

only peak systolic velocities obtained from the TO method when compared with TEE have an acceptable percentage error of $23.4 \%$, which is less than the expected LOA of $25.8 \%$. The corresponding $r$ value of 0.85 showed likewise a strong correlation (Figure 5). $\mathrm{TO}_{\mathrm{Q} \text { Vmean }}$ compared with PACTD also had a percentage error below the expected LOA, but above $30 \%$, which is recommended as the limit for interchangeability by Critchley et al. ${ }^{29}$

According to Table 1 and 2, TO performs less accurately when applied intraoperatively on the ascending aorta than on the flowrig. Probably, this is a consequence of the complex flow present in the ascending aorta. As illustrated in Figure 1, several patients had systolic backflow, and the flow during late systole and diastole is highly complex, as previously reported..$^{22,23,31}$ Therefore, velocity estimation with $2 \mathrm{D}$ vector velocity US of the ascending aorta is liable to underestimate the blood flow as the highest velocities can be found off-plane.

TO had a mean bias of $12.6 \mathrm{~cm} / \mathrm{s}$ compared with TEE corresponding to an average underestimation of $11.8 \%$ comparable with previous results. ${ }^{16,19,23}$ Apart from the inherent bias in the estimation scheme, Pedersen et al. argue the temporal resolution of 16 frames/s also adds up to the underestimation, as peak systolic velocities are missed out. ${ }^{19}$ 


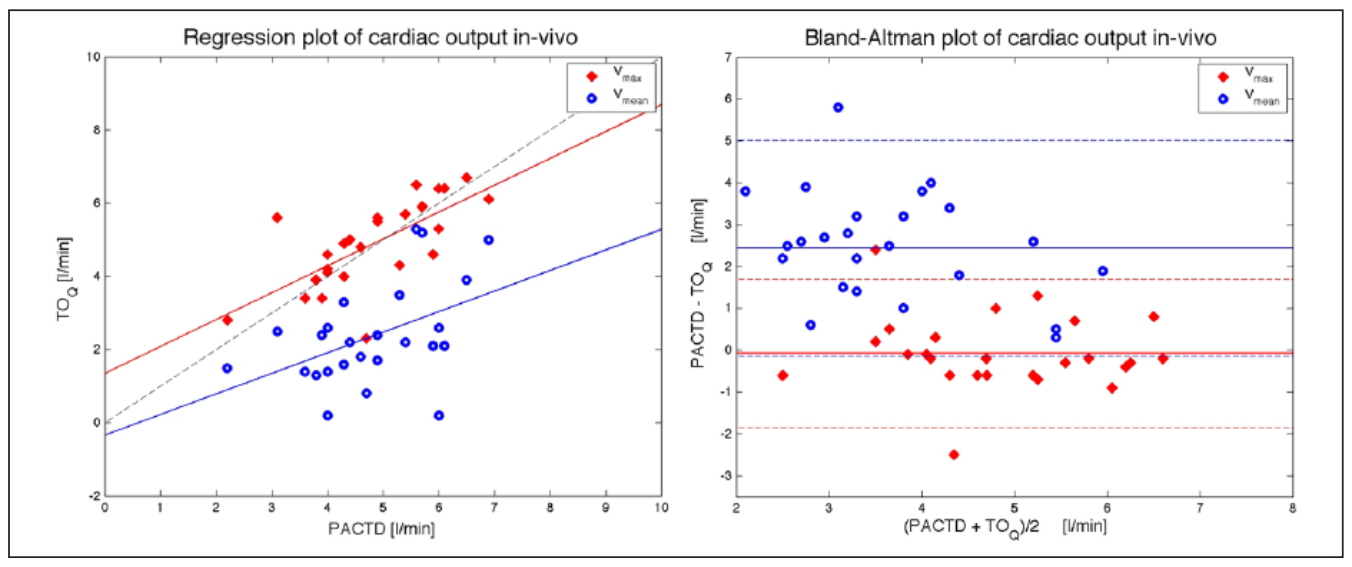

Figure 6. Cardiac output estimated with TO and PACTD in vivo and evaluated with Bland-Altman and regression plots. $\mathrm{TO}=$ transverse oscillation; PACTD = pulmonary artery catheter thermodilution.

The correlation between TO and PACTD was strong when using Vmax $(r=0.70)$ but weak when using Vmean $(r=0.46)$. TO was significantly less precise than PACTD for both Vmax and Vmean, and both approaches were rejected when evaluating for percentage error. Thus, the data indicate that TO is not interchangeable with PACTD, which supports previously preliminary published results. ${ }^{22,23}$

Using Vmean for flow rate estimation is the correct approach for a complex flow environment such as the ascending aorta, as only the assumption of axisymmetrical flow is made. However, a substantial underestimation compared with PACTD was found (Table 2), and apart from the inherent velocity underestimation and inadequate PRF settings, probably the non-laminar nature of the blood flow was the most important confounder. Hansen et al. showed that calculated stroke volume from 2D blood velocity estimates in the carotid artery among 11 healthy volunteers had a mean variation of $24.1 \%$ for different angles, mainly due to asymmetric flow. ${ }^{18}$

The flowrig was employed with flow rates from 100 to $1550 \mathrm{ml} / \mathrm{min}$ (Figures 2 to 4). Thus, physiological flow rates, which can be as high as $201 /$ min during systole, that is, the peak flow rate, were not reached in the flowrig. Therefore, the examined performance of TO in flowrig does not include flow rates found in the ascending aorta, as these flow rates are not reachable in the flow phantom. However, physiological velocities in the flowrig were achieved with $105 \mathrm{~cm} / \mathrm{s}$, and thus, the main difference between the flowrig and the in vivo setup was the dimensions of the vessels. In the flowrig, a tube of $8 \mathrm{~mm}$ was used, while the average diameter of the ascending aorta was $31.16 \mathrm{~mm}(S D=4.07)$.

The correction scheme for flow rates is used under the assumption that the systematic bias for TO found in the flowrig experiment is valid at the higher flow rates found in vivo, which are created by a larger diameter of the ascending aorta compared with the tube in the flowrig. Thus, some of the bias found in the in vivo experiment for TO compared with PACTD may represent an estimation bias of the TO method at these high physiological flow rates.

According to Table 2 and Figure 6, Vmax used for in vivo flow rate estimation resulted in a better performance than using Vmean. So even though the assumption of parabolic flow profile is not met, the overestimation when assuming parabolic flow seems to compensate for the underestimation when estimating flow rate of complex flow with a 2D US method. This is in concordance with the preliminary results of intraoperatively vector flow imaging of the aortic flow previously published. ${ }^{23}$

The bias found in vivo when using TO is not systematic (Figures 5 and 6). Therefore, the calculated correction found in the flowrig for the underestimation of velocities and flow rates due 
to the bias in the TO estimation scheme and the PRF limitation of TO did not improve percentage errors for the comparisons (Table 2).

Hansen et al. showed that stroke volume estimated with TO compared with MRI performed with a mean bias of $5 \%$, a percentage error of $29 \%$, and an $r$ value of 0.91 , thus, a slightly better agreement with the reference method than found in this study. ${ }^{17,18}$ First, the stroke volume was estimated in the carotid artery, where the flow is less complex than in the ascending aorta. Second, in the previous study, TO was implemented on an experimental scanner, while in the present study, TO is implemented on a commercial scanner. This implies that post-processing in the present study is less flexible, for example, wall filtering adjustments.

The default wall filter setting for TO scans was used in this study. The wall filter in default mode changes automatically whenever the PRF setting is changed. Patients with high systolic velocities were examined with a high PRF setting of $11.4 \mathrm{kHz}$ and a wall filter of $571 \mathrm{~Hz}$ going down to a PRF of $7.0 \mathrm{kHz}$ with a wall filter of $348 \mathrm{~Hz}$ for patients with lower systolic velocities.

Slower flow components along the vessel wall are liable to be missed out with a high wall filter setting. When using Vmean for the volume flow calculation, where all flow components of the lumen are used, the wall filtering can create an underestimation of volume flow. A solution is adaptive filtering with a wall filter adapting to the changing flow. It is difficult to implement in real time, but has previously been used off-line with the vector velocity method directional beamforming. ${ }^{17}$

In a recent study with TO implemented as in this study, Hansen et al. found an underestimation of TO derived volume flow in arteriovenous fistulas of 30\% compared with UDT but with a significantly higher precision when compared with UDT. ${ }^{24}$ The flow of the fistula is more laminar and parabolic, and less pulsatile and complex than flow of the ascending aorta, thus, probably better suited for volume flow estimation with TO using 2D US and more comparable with the TO performance in the flowrig experiments reported in this study.

Exact volume flow estimation is crucial in the surgical setup as well as in the clinical practice with heart-related diseases. However, all 2D US methods for flow estimation are hampered by the lacking third dimension, when flow estimations are done on complex in vivo blood flow. A recent study showed that volume flow estimation obtained with TEE had a percentage error of $38.6 \%$ compared with PACTD, with the conclusion that TEE and PACTD are not interchangeable. ${ }^{30}$ Depending on the approach used for volume flow estimation from either Vmax or Vmean obtained with TEE, assumptions of parabolic and axisymmetrical flow patterns are necessary, and as shown in this study, these assumptions create major biases. Whether vector flow imaging or TEE is used, the only solution for volume flow estimation in aorta is to expand the US flow examination to cover all three dimensions. This has been achieved with TO on the experimental scanner SARUS ${ }^{32}$ with both flowrig and in vivo experiments. ${ }^{33}$

For both TO and TEE, the velocities are only estimated in-plane. It is impossible to cover identical planes with the two methods. Therefore, in the comparison of peak systolic velocity estimation with TO and TEE, there is an assumption of axisymmetrical flow, although only concerning the estimated peak systolic velocities. This assumption adds to the bias of the comparison between the two methods.

Apart from the limitation of 2D US, also the PRF is a challenge for TO estimation. One solution to the PRF limitation is to gate the TO estimation with electrocardiography, record in two sessions with low and high PRF settings, and off-line to calculate the volume flow by adding diastolic and systolic vector flow estimates. Another approach is to obtain the vector velocities with, for example, the synthetic aperture method, where recursive velocity estimation is possible due to a continuous availability of data. ${ }^{34}$ Accordingly, a low and high PRF setting can be achieved simultaneously resulting in improved velocity estimation as shown by Hoyos et al. ${ }^{35} \mathrm{~A}$ 
similar approach could theoretically also be realized with TO, if only one line of vector velocities is measured repeatedly as in spectral Doppler instead of the present setup, where 2D vector flow images are interleaved with B-mode images.

In this study, all measurements in comparison were acquired within $5 \mathrm{~min}$ and, therefore, some variation must be expected. This can explain a part of the difference between the TO and the reference methods.

Also, the inaccuracies of the reference methods were important confounders. In this study, the mean peak systolic velocity in the ascending aorta estimated with TEE was $112 \mathrm{~cm} / \mathrm{s}$ (range: 80 $\mathrm{cm} / \mathrm{s}-180 \mathrm{~cm} / \mathrm{s}$; Figure 5), which is comparable with another study using US. ${ }^{36}$ According to a MRI study, the peak systolic velocity in normal participants is approximately $80 \mathrm{~cm} / \mathrm{s} .{ }^{37}$ Conventional spectral Doppler is, like TO, limited to 2D and has a tendency to overestimate velocities in the range from $5 \%$ to $47 \%$ when compared with other modalities. ${ }^{38-41}$ In a recent comparison study with two vector velocity methods, conventional spectral Doppler overestimated velocities due to spectral broadening. ${ }^{10}$ Likewise, studies have shown that PACTD has a tendency to overestimate cardiac output compared with flowrig and aortic flow probe with mean errors from $10 \%$ to $39 \% .{ }^{42,43}$

It is expected that the flow profile in the flowrig is not fully developed, due to an insufficient entrance length of less than $2 \mathrm{~m}$. This could explain why flow rates found with Vmax are more underestimated than with Vmean, and this may result in a correction for Vmax, which covers both limitations of the TO method and the flowrig (Table 2). Furthermore, the flow is not measured by a flowmeter but is taken from the pump settings. According to the manufacturer of the flow pump, the flow rates reported have SDs of less than $2 \%$.

According to simulations, the TO method has an almost constant relative bias with insonation angles between $60^{\circ}$ and $90^{\circ}$, whereas the relative $S D$ is reduced from $7 \%$ to $5 \%$ when increasing the insonation angle from $60^{\circ}$ to $90^{\circ} . .^{25}$ As the average beam-to-flow angle in vivo was $76.3^{\circ}$ compared with $90^{\circ}$ in the flowrig, a slightly higher variation with an almost identical bias of the vector velocities was expected in vivo when compared with the flowrig measurements.

The average Doppler gain and PRF setting were different in the flowrig and in vivo experiments. However, according to the flowrig measurements, a strong linear systematic bias in TO was present for both constant and pulsatile flow. Thus, a higher flow rate in the flowrig using a PRF setting resembling the in vivo setup would not have changed the result. The Doppler gain was adjusted so the flow signal filled the vessel without blooming artefacts. As the flow patterns in the flowrig and in vivo were different, the average gain was slightly lower in vivo. The effect of the gain setting of TO has not been assessed previously, and is not evaluated in this study.

The reported incidence of serious complications related to epicardial and epiaortic echocardiography as well as contraindications are virtually nonexistent, ${ }^{44}$ while TEE is contraindicated by several esophageal problems, for example, previous esophagectomy, esophagogastrectomy, esophageal stricture, tracheoesophageal fistula, postesophageal surgery, and esophageal trauma. ${ }^{1}$ In this study, no complications during the epiaortic scans were observed, and all enrolled patients were scanned. However, epiaortic scan has certain limitations. When epiaortic scans are performed, the surgeons are not able to perform the surgery, thus, the epiaortic TO scans prolonged the time in surgery for approximately $5 \mathrm{~min}$, while scanning with TEE was achievable throughout the entire surgery without delay. Furthermore, for a few patients, the anatomy and the sternotomy dictated a transducer placement slightly more downstream of the valve than in the majority of the patients.

Even though vector flow imaging in this study is used epiaortically, the future goal is to use the TO method in transthoracic echocardiography and in TEE. TO was employed intraoperatively to examine the heart, as the scan depth is limited to $5 \mathrm{~cm}$ with the current transducer setup, but transthoracic echocardiography with TO is achievable with both curved and phased array 
transducers, as shown in preliminary studies. ${ }^{27,45}$ Thereby, new insonation windows of the heart will be possible due to the angle-independency in vector velocity estimation, and new information of the complex flow patterns of the cardiovascular system can be obtained as previous preliminary studies have indicated..$^{22,23}$

\section{Conclusion}

The vector velocity method TO is an accurate and reliable US method for velocity and flow rate estimation in flowrig for both constant and pulsatile flow using velocities from 5 to $105 \mathrm{~cm} / \mathrm{s}$ and flow rates from 100 to $1550 \mathrm{ml} / \mathrm{min}$. The systematic bias due to the inherent underestimation of vector velocities as well as the PRF limitation of TO was corrected and resulted in significantly improved percentage errors.

Twenty-five patients were scanned intraoperatively during cardiac surgery with TO and compared with estimates of TEE and PACTD. TO estimates correlated to both estimates of TEE and PACTD. The precision of TO was comparable with TEE but significantly reduced compared with PACTD, and only the percentage error for TO compared with TEE was below the expected LOA. Applying the flowrig-derived correction for systematic bias slightly improved the in vivo TO flow velocity estimation but not flow rate estimation, as the main confounder of aortic flow estimation using 2D US is flow complexicity.

Thus, this study indicates that TO is a reliable angle-independent US alternative for cardiac velocity estimation when compared with conventional spectral Doppler, but is not with the present setup interchangeable with PACTD for cardiac volume flow estimation.

TO is a promising method for cardiac flow examinations during cardiac or major non-cardiac surgery. However, to become an alternative to conventional Doppler US and PACTD, the precision should be improved and the bias reduced for TO. This may be achieved by expanding TO to three-dimensional (3D) US, and by improving the TO method in terms of inherent bias, PRF dependency, and temporal resolution.

\section{Declaration of Conflicting Interests}

The author(s) declared no potential conflicts of interest with respect to the research, authorship, and/or publication of this article.

\section{Funding}

The author(s) disclosed receipt of the following financial support for the research, authorship, and/or publication of this article: The study was supported by grant number 82-2012-4 from The Danish National Advanced Technology Foundation and by BK Medical ApS.

\section{References}

1. American Society of Anesthesiologists and Society of Cardiovascular Anesthesiologists Task Force on Transesophageal Echocardiography. Practice guidelines for perioperative transesophageal echocardiography. An updated report by the American Society of Anesthesiologists and the Society of Cardiovascular Anesthesiologists Task Force on Transesophageal Echocardiography. Anesthesiology. 2010;112(5):1084-96.

2. Akiyama K, Arisawa S, Ide M, Iwaya M, Naito Y. Intraoperative cardiac assessment with transesophageal echocardiography for decision-making in cardiac anesthesia. Gen Thorac Cardiovasc Surg. 2013;61(6):320-9.

3. Szabo TL. Diagnostic Ultrasound Imaging: Inside Out. Oxford: Elsevier; 2014.

4. American Society of Anesthesiologists Task Force on Pulmonary Artery Catheterization. Practice guidelines for pulmonary artery catheterization: an updated report by the American Society of 
Anesthesiologists Task Force on Pulmonary Artery Catheterization. Anesthesiology. 2003;99(4):9881014.

5. Sandham JD, Hull RD, Brant RF, Knox L, Pineo GF, Doig CJ, et al. A randomized, controlled trial of the use of pulmonary-artery catheters in high-risk surgical patients. N Engl J Med. 2003;348(1):5-14.

6. Bonnefous O. Measurement of the complete (3D) velocity vector of blood flows. Proc IEEE Ultrason Symp. 1988:795-9.

7. Fox MD. Multiple crossed-beam ultrasound Doppler velocimetry. IEEE Trans Son Ultrason. 1978;25:281-6.

8. Newhouse VL, Censor D, Vontz T, Cisneros JA, Goldberg BB. Ultrasound Doppler probing of flows transverse with respect to beam axis. IEEE Trans Biomed Eng. 1987;34:779-88.

9. Trahey GE, Allison JW, Ramm OT. Angle independent ultrasonic detection of blood flow. IEEE Trans Biomed Eng. 1987;34(12):965-7.

10. Tortoli P, Lenge M, Righi D, Ciuti G, Liebgott H, Ricci S. Comparison of carotid artery blood velocity measurements by vector and standard Doppler approaches. Ultrasound Med Biol. 2015;41(5):1354-62.

11. Lovstakken L, Ibrahim KS, Vitale N, Henriksen ST, Kirkeby-Garstad I, Torp H, et al. Blood flow imaging: a new two-dimensional ultrasound modality for enhanced intraoperative visualization of blood flow patterns in coronary anastomoses. J Am Soc Echocardiogr. 2008;21(8):969-75.

12. Lovstakken L, Nyrnes SA, Haugen BO, Torp H. Angle-independent quantification of complex flow patterns in congenital heart disease. In: IEEE International Ultrasonics Symposium, Orlando, FL, 1821 October 2011, pp. 1246-9.

13. Nyrnes SA, Lovstakken L, Torp H, Haugen BO. Blood flow imaging - a new angle-independent ultrasound modality for the visualization of flow in atrial septal defects in children. Echocardiography. 2007;24(9):975-81.

14. Jensen JA, Munk P. A new method for estimation of velocity vectors. IEEE Trans Ultrason Ferroelec Freq Contr. 1998;45:837-51.

15. Jensen JA. A new estimator for vector velocity estimation. IEEE Trans Ultrason Ferroelec Freq Contr. 2001;48:886-94.

16. Udesen J, Jensen JA. Investigation of transverse oscillation method. IEEE Trans Ultrason Ferroelec Freq Contr. 2006;53:959-71.

17. Hansen KL, Udesen J, Oddershede N, Henze L, Thomsen C, Jensen JA, et al. In vivo comparison of three ultrasound vector velocity techniques to MR phase contrast angiography. Ultrasonics. 2009;49(8):659-67.

18. Hansen KL, Udesen J, Thomsen C, Jensen JA, Nielsen MB. In vivo validation of a blood vector velocity estimator with MR angiography. IEEE Trans Ultrason Ferroelec Freq Contr. 2009;56(1):91-100.

19. Pedersen MM, Pihl MJ, Haugaard P, Hansen JM, Hansen KL, Nielsen MB, et al. Comparison of realtime in vivo spectral and vector velocity estimation. Ultrasound Med Biol. 2012;38(1):145-51.

20. Liebgott H, Basarab A, Gueth P, Friboulet D, Delachartre P. Transverse oscillations for tissue motion estimation. Ultrasonics. 2010;50(6):548-55.

21. Varray F, Liebgott H. Multi-resolution transverse oscillation in ultrasound imaging for motion estimation. IEEE Trans Ultrason Ferroelec Freq Contr. 2013;60(7):1333-42.

22. Hansen KL, Pedersen MM, Moller-Sorensen H, Kjaergaard J, Nilsson JC, Lund JT, et al. Intraoperative cardiac ultrasound examination using vector flow imaging. Ultrason Imaging. 2013;35(4):318-32.

23. Hansen KL, Moller-Sorensen H, Pedersen MM, Hansen PM, Kjaergaard J, Lund JT, et al. First report on intraoperative vector flow imaging of the heart among patients with healthy and diseased aortic valves. Ultrasonics. 2015;56:243-50.

24. Hansen PM, Olesen JB, Pihl MJ, Lange T, Heerwagen S, Pedersen MM, et al. Volume flow in arteriovenous fistulas using vector velocity ultrasound. Ultrasound Med Biol. 2014;40(11):2707-14.

25. Pihl MJ, Svetoslav N, Haugaard P, Hemmsen MC, Jensen JA. Performance of the Transverse Oscillation Method using Beamformed Data from a Commercial Scanner. In: IEEE International Ultrasonics Symposium, Rome, 20-23 September 2009, pp. 1-4.

26. Reeves ST, Glas KE, Eltzschig H, Mathew JP, Rubenson DS, Hartman GS, et al. Guidelines for performing a comprehensive epicardial echocardiography examination: recommendations of the American Society of Echocardiography and the Society of Cardiovascular Anesthesiologists. J Am Soc Echocardiogr. 2007;20(4):427-37. 
27. Jensen JA. Optimization of transverse oscillating fields for vector velocity estimation with convex arrays. Proc IEEE Ultrason Symp. 2013;1753-6.

28. Altman DG. Practical Statistics for Medical Research. London: Chapmann \& Hall/CRC; 1991.

29. Critchley LA, Critchley JA. A meta-analysis of studies using bias and precision statistics to compare cardiac output measurement techniques. J Clin Monit Comput. 1999;15(2):85-91.

30. Moller-Sorensen H, Graeser K, Hansen KL, Zemtsovski M, Sander EM, Nilsson JC. Measurements of cardiac output obtained with transesophageal echocardiography and pulmonary artery thermodilution are not interchangeable. Acta Anaesthesiol Scand. 2014;58(1):80-8.

31. Bensalah M, Bollache E, Kachenoura N, Giron A, De Cesare A, Macron L, et al. Geometry is a major determinant of flow reversal in proximal aorta. Am J Physiol Heart Circ Physiol. 2014;306(10): 1408-16.

32. Jensen JA, Holm O, Jensen LJ, Bendsen H, Nikolov S, Tomov BG, et al. Ultrasound research scanner for real-time synthetic aperture image acquisition. IEEE Trans Ultrason Ferroelec Freq Contr. 2005;52(5):881-91.

33. Pihl MJ, Stuart MB, Tomov BG, Rasmussen MF, Jensen JA. A transverse oscillation approach for estimation of three-dimensional velocity vectors, part II: experimental validation. IEEE Trans Ultrason Ferroelectr Freq Control. 2014;61(10):1608-18.

34. Oddershede N, Jensen JA. Effects influencing focusing in synthetic aperture vector flow imaging. IEEE Trans Ultrason Ferroelec Freq Contr. 2007;54:1811-25.

35. Hoyos CAV, Stuart MB, Jensen JA. Increasing the dynamic range of synthetic aperture vector flow imaging. Proc SPIE Med Imag. 2014;9040.

36. Gisvold SE, Brubakk AO. Measurement of instantaneous blood-flow velocity in the human aorta using pulsed Doppler ultrasound. Cardiovasc Res. 1982;16(1):26-33.

37. Bogren HG, Buonocore MH. 4D magnetic resonance velocity mapping of blood flow patterns in the aorta in young vs. elderly normal subjects. J Magn Reson Imaging. 1999;10(5):861-9.

38. Hoskins PR. Accuracy of maximum velocity estimates made using Doppler ultrasound systems. $\mathrm{Br} \mathrm{J}$ Radiol. 1996;69(818):172-7.

39. Jiang J, Strother C, Johnson K, Baker S, Consigny D, Wieben O, et al. Comparison of blood velocity measurements between ultrasound Doppler and accelerated phase-contrast MR angiography in small arteries with disturbed flow. Phys Med Biol. 201121;56(6):1755-73.

40. Park MY, Jung SE, Byun JY, Kim JH, Joo GE. Effect of beam-flow angle on velocity measurements in modern Doppler ultrasound systems. AJR Am J Roentgenol. 2011;198(5):1139-43.

41. Walker A, Olsson E, Wranne B, Ringqvist I, Ask P. Accuracy of spectral Doppler flow and tissue velocity measurements in ultrasound systems. Ultrasound Med Biol. 2004;30(1):127-32.

42. Yang XX, Critchley LA, Joynt GM. Determination of the precision error of the pulmonary artery thermodilution catheter using an in vitro continuous flow test rig. Anesth Analg. 2011;112(1):70-7.

43. Yang XX, Critchley LA, Rowlands DK, Fang Z, Huang L. Systematic error of cardiac output measured by bolus thermodilution with a pulmonary artery catheter compared with that measured by an aortic flow probe in a pig model. J Cardiothorac Vasc Anesth. 2013;27(6):1133-9.

44. Eltzschig HK, Kallmeyer IJ, Mihaljevic T, Alapati S, Shernan SK. A practical approach to a comprehensive epicardial and epiaortic echocardiographic examination. J Cardiothorac Vasc Anesth. 2003;17(4):422-9.

45. Pihl MJ, Marcher J, Jensen JA. Phased-array vector velocity estimation using transverse oscillations. IEEE Trans Ultrason Ferroelectr Freq Control. 2012;59(12):2662-75. 